\title{
Classifiers for centrality determination in proton-nucleus and nucleus-nucleus collisions
}

\author{
Igor Altsybeev ${ }^{1, a}$ and Vladimir Kovalenko ${ }^{1, b}$ \\ ${ }^{1}$ Saint-Petersburg State University, 7/9 Universitetskaya nab., St. Petersburg, 199034 Russia
}

\begin{abstract}
Centrality, as a geometrical property of the collision, is crucial for the physical interpretation of nucleus-nucleus and proton-nucleus experimental data. However, it cannot be directly accessed in event-by-event data analysis. Common methods for centrality estimation in A-A and p-A collisions usually rely on a single detector (either on the signal in zero-degree calorimeters or on the multiplicity in some semi-central rapidity range). In the present work, we made an attempt to develop an approach for centrality determination that is based on machine-learning techniques and utilizes information from several detector subsystems simultaneously. Different event classifiers are suggested and evaluated for their selectivity power in terms of the number of nucleons-participants and the impact parameter of the collision. Finer centrality resolution may allow to reduce impact from so-called volume fluctuations on physical observables being studied in heavy-ion experiments like ALICE at the LHC and fixed target experiment NA61/SHINE on SPS.
\end{abstract}

\section{Introduction}

Machine-learning (ML) techniques have been used in High-Energy Physics (HEP) so far in a limited number of ways. The most common application of the ML is a search for rare processes (for example, boosted decision trees were used in searches for $B_{s}^{0} \rightarrow \mu^{+} \mu^{-}$decay [1]). Another use case is a trigger optimization [2]. In experiments dedicated to studies of heavy-ion collisions, like ALICE at the LHC, ML is used mainly for detector response optimization, as described in overview [3]. For example, the Bayesian approach is used to more effectively combine the particle identification capabilities of various detectors [4].

It is interesting to find new applications of ML for physics analysis in experiments of heavy-ion collisions. In the present work, we tried to address the task of the centrality determination. The centrality is a key parameter in the study of the properties of QCD matter at extreme temperature and energy density, because it is directly related to the initial overlap region of the colliding nuclei [5]. The centrality is usually expressed as a percentage of the total nuclear interaction cross section $\sigma$ : $c=\int_{0}^{b} \frac{d \sigma}{d x} d x$, where impact parameter $(b)$ is the distance between the centers of the two colliding nuclei. Taking the full impact parameter range as $0-100 \%$, peripheral collisions have centrality closer to $100 \%$, while the most central events are close to $0 \%$.

\footnotetext{
a e-mail: i.altsybeev@ spbu.ru

be-mail: v.kovalenko@spbu.ru
} 
Impact parameter $b$ is not directly accessible in experiment. In order to obtain estimation of centrality experimentally, a signal distribution in some detecting system is usually used: the distribution is divided into centrality classes, which are then related to some interval of the impact parameter, with some estimated resolution. To make this relation, the geometrical Glauber model [6] is usually used, which treats a nuclear collision as a superposition of binary nucleon-nucleon interactions. A nucleon that undergoes one or more collisions with nucleons of the other nucleus, is usually called a participant nucleon. The volume of the initial overlap region is expressed via the number of participant nucleons $N_{\text {part }}$. A number of binary nucleon-nucleon collisions is usually denoted as $N_{\text {coll }}$. A number of spectator nucleons $N_{\text {spec }}$ constitutes the part of the nuclear volume not involved in the interaction.

Any increase in centrality resolution would be beneficial for certain types of physics analysis. For example, higher centrality resolution is desirable in class $0-1 \%$ of $\mathrm{Pb}-\mathrm{Pb}$ collisions to select the most central events, where double-peaked structure was observed in azimuthal profile of two-particle correlation function [7].

The present exploratory work is dedicated to ML-based task for centrality determination. The aim is to select "centrality classes" with improved resolution in terms of the impact parameter $b$ in $\mathrm{Pb}$ $\mathrm{Pb}$ and $N_{\text {part }}$ in $\mathrm{p}-\mathrm{Pb}$ collisions using signals from several subsystems of the detector simultaneously. The task is performed using simulated events. Dealing with detector-induced losses in efficiency, contamination by secondary particles and application of this technique to real data are out of the scope of this article.

\section{Centrality determination in ALICE experiment}

In the ALICE experimental setup [8], the most commonly used detecting system for centrality determination is a pair of so-called VZERO detectors, two forward scintillator arrays with coverage $-3.7<\eta<-1.7$ (VZERO-C) and $2.8<\eta<5.1$ (VZERO-A). Since these are non-tracking detectors, signal in each VZERO is proportional to charged particle multiplicity of primary tracks and secondary tracks from weak decays and detector material. The summed signal in both VZERO scintillators is usually denoted as V0M. Distribution of the VOM amplitude shown in figure 1 (a) is fitted with the

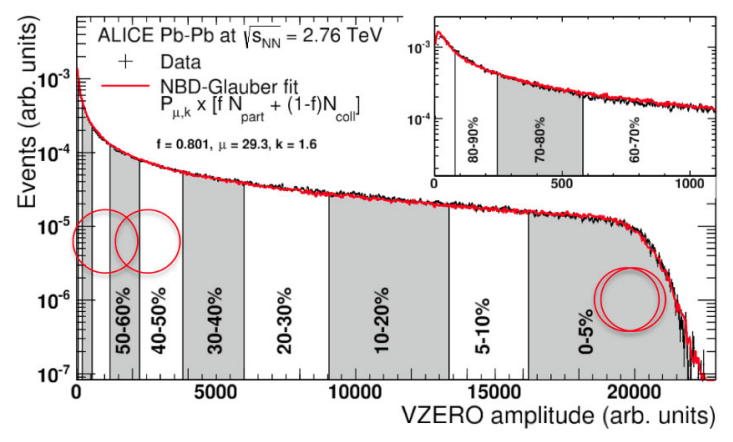

(a)

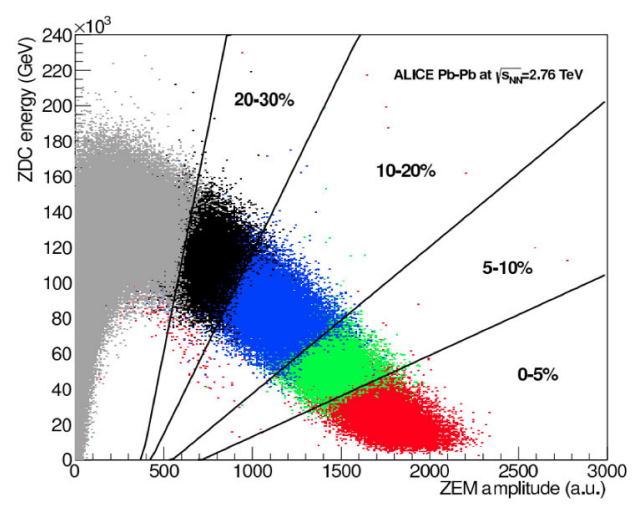

(b)

Figure 1. (a): Distribution of the sum of amplitudes in the VZERO scintillators (VOM). The distribution is fitted with the NBD-Glauber fit shown as a line, obtained centrality classes are indicated. (b): Spectator energy deposited in the ZDCs as a function of ZEM amplitude [5]. 
Glauber model coupled with negative binomial distribution (NBD), and divided into centrality classes [5]. Values of $N_{\text {part }}$ and $N_{\text {coll }}$ in each centrality class are extracted from the fit.

Another set of detectors used for centrality determination are the Zero-Degree Calorimeters (ZDC) placed at $\approx \pm 110 \mathrm{~m}$ in the LHC tunnel. The energy deposited in the ZDCs is directly related to the number of spectator nucleons $N_{\text {spec }}$. Combining signal from ZDCs with the energy measured by small EM calorimeters (ZEM) placed at A-side of ALICE at $4.8<\eta<5.7$, a two-dimensional distribution can be plotted (figure 1, b). The centrality classes are determined from this plot by splitting it with lines, which are assumed to intersect at some common point [5].

Charged primary particles are reconstructed in ALICE with the central barrel detectors combining information from the Inner Tracking System (ITS) and the Time Projection Chamber (TPC). The TPC, together with the ITS, provides charged particle momentum measurement, particle identification and vertex determination. Both detectors are located inside the $0.5 \mathrm{~T}$ solenoidal field and have full azimuthal coverage for track reconstruction within a pseudo-rapidity window of $|\eta|<0.8$.

\section{$3 \mathrm{ML}$ task for simulated $\mathrm{Pb}-\mathrm{Pb}$ events in the conditions of ALICE experiment}

The AMPT Monte Carlo event generator [9] was used to simulate $\mathrm{Pb}-\mathrm{Pb}$ collisions at $\sqrt{s_{\mathrm{NN}}}=$ $2.76 \mathrm{TeV}$. The simulation contains no detector response, so "signals" of the real detector were emulated directly by taking generated particles in certain areas of the phase space. Five main features were selected for the ML task in correspondence with the subsystems of the ALICE detector (figure 2). These are multiplicities of charged particles obtained in AMPT simulation within acceptances of each of the VZERO scintillators (A, C) and the TPC, and also numbers of neutrons-spectators, which in the "ideal world" would be measured in each of the ZDCs (A and C). Also, three additional features were introduced in order to study their importance for improvement of the final centrality resolution: average transverse momentum of particles in the TPC within each event $\left(\left\langle p_{\mathrm{T}}\right\rangle\right)$, kaon-to-pion $(\mathrm{K} / \pi)$ and proton-to-pion $(\mathrm{p} / \pi)$ ratios in each event. These observables are known to be dependent on centrality of heavy-ion collisions and possibly possess some discriminative power.

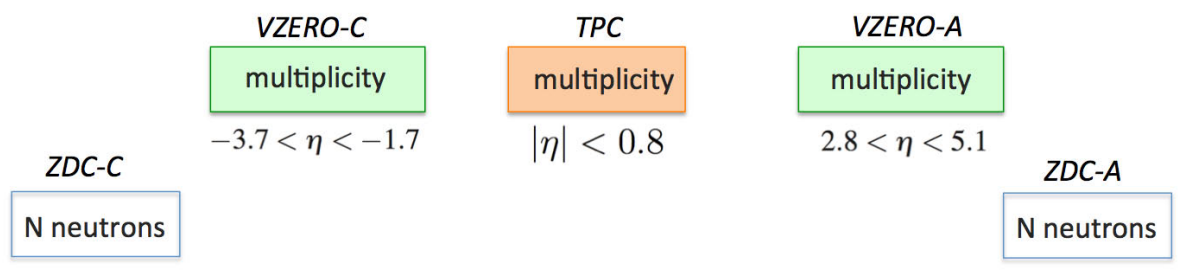

Figure 2. Detecting systems, from which five features for the ML task were taken: multiplicities in acceptances of VZERO-A, C, TPC, and numbers of nucleons-spectators in each of two ZDCs.

\subsection{Regression task}

It is interesting to find out whether we have some improvement in centrality resolution if, instead of V0M, we perform event selection for centrality classes using ML regressor. To perform ML regression task for centrality determination, TMVA package [10] of version 4.2.0 was used. For this task, a feature set of five variables, described above, was taken, with the impact parameter $b$ as a target for 
regression. Among several probed regressors, good performance was obtained, in particular, with a Boosted Decision Tree with gradient boosting (denoted as BDTG), results from which are used here for illustration. Booking in TMVA was done with the following line:

factory->BookMethod( TMVA: :Types: :kBDT, "BDTG", "!H:!V:NTrees=2000: :BoostType=Grad:

Shrinkage $=0.1$ :UseBaggedBoost $:$ BaggedSampleFraction $=0.5:$ nCuts $=20:$ MaxDepth= $3:$ MaxDepth $=4 ")$;

To focus on more central collisions, events for the analysis were preselected using V0M estimator to be within $0-10 \%$ centrality class (see centrality classes in figure 1 , a). This preselected set contains $400 \mathrm{k}$ of events, and it was randomly split into two halves: 200k events for training and 200k for testing.

Distribution of the truth impact parameter $b$ in events, preselected by V0M, is shown in figure 3 as a blue shaded area. Output from BDTG regressor, which is a distribution of estimated values of $b$, is shown on the same plot in red. It is "squeezed" from both left and right sides, demonstrating inability of the regressor to reach left and right extremes of the truth distribution.

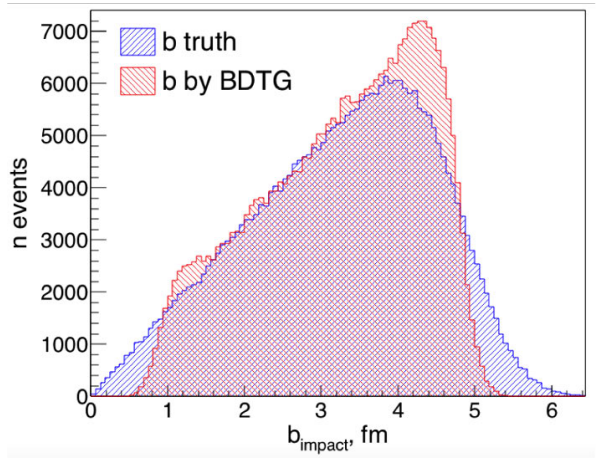

Figure 3. Distribution of impact parameter $b$ for events in $0-10 \%$ centrality class preselected by simulated V0M signal (wide distribution, shown in blue). Output from BDTG regressor with impact parameter $b$ as a target is narrower and is shown in red.

In order to use BDTG regressor as another (alternative) centrality estimator, its output was split into "centrality bins" in the same manner as it is done for conventional V0M distribution (recall figure 1, a). To compare performance of the new BDTG estimator with the V0M in terms of impact parameter resolution, outputs from both estimators were split into 10 centrality bins (this was done for $0-10 \%$ preselected by VOM events, so obtained classes are of $1 \%$ width). Figure 4 shows truth impact parameter distributions for events within $0-1 \%, 1-2 \%$ and $2-3 \%$ centrality classes selected by

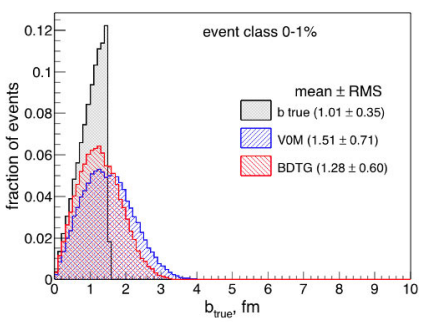

(a)

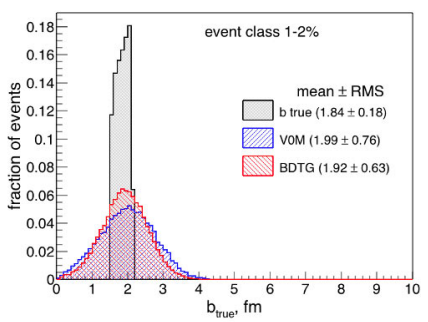

(b)

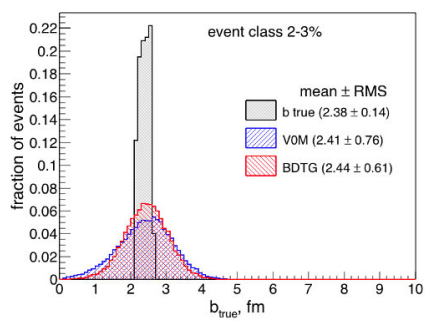

(c)

Figure 4. Distributions of impact parameter within centrality classes $0-1,1-2$ and 2-3\% selected by V0M and BDTG estimators (color code is different from figure 3). For comparison, grey distributions show slices of $1 \%$ width when slicing into centrality classes is done by truth $b$ itself. 
V0M (in blue) and selected using new BDTG estimator (in red). It can be seen that when selection is done with the BDTG estimator, distributions of $b$ are narrower than with V0M selection, which means higher resolution in terms of $b$. Also, in most central 0-1\% event class selected with the BDTG estimator, the $b$-distribution goes closer towards $b=0$ (figure 4 , a), indicating that within this class we indeed select more central events than with V0M.

The same information is summarized in figure 5, where each colored region covers the mean values of $b$ distributions \pm their RMS in centrality classes of $1 \%$-width in $0-10 \%$ range: the red area stands for centrality selection by the BDTG estimator, and it is narrower than the green area for selection using VOM, especially for the most central events. For comparison, the blue area is for the case when slicing into centrality classes is done by truth $b$ itself.

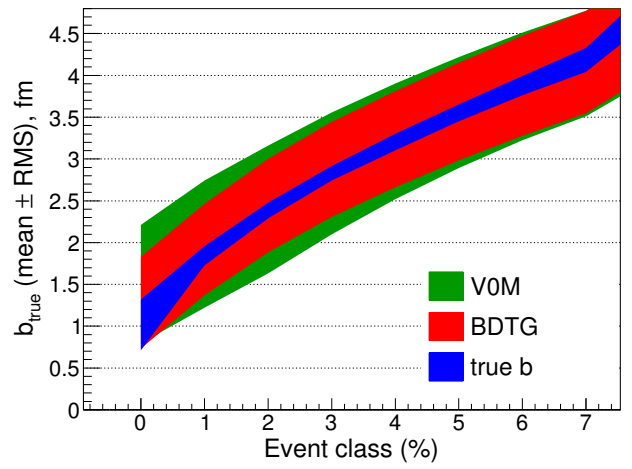

Figure 5. Mean \pm RMS of impact parameter $b$ distributions for events within centrality classes of $1 \%$ width, which are selected using simulated multiplicity distribution in VOM acceptance (in green) and BDTG (in red) estimators. Blue area covers mean \pm RMS of $b$ distributions, when slicing into centrality classes is done by truth $b$ itself.

\subsection{Classification task for the most central events}

In this section, centrality determination task is considered as a classification problem. Namely, within $0-10 \%$ class of events, preselected with simulated multiplicity distribution in VZERO acceptance,

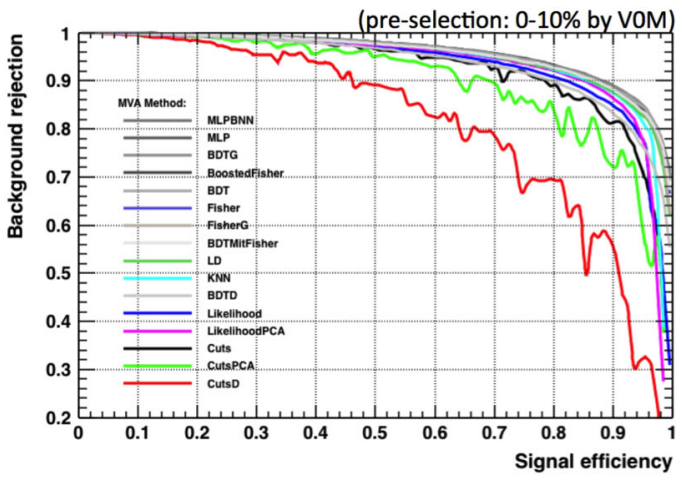

(a)

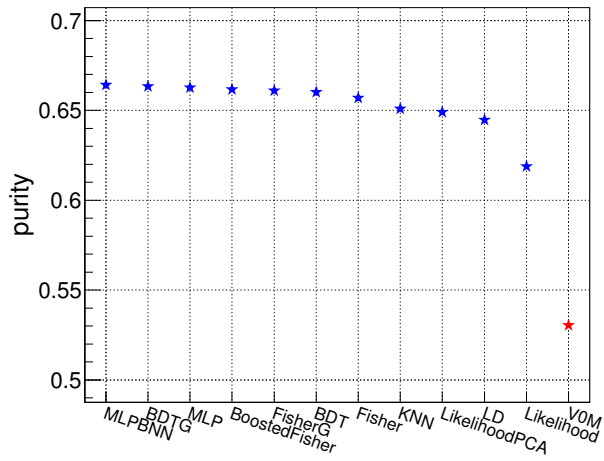

(b)

Figure 6. (a): Signal efficiency $v s$ background rejection for a set of classifiers (TMVA). (b): Purities reached with classifiers in $0-1 \%$ centrality class in terms of impact parameter. Red star is for purity of $0-1 \%$ event class with V0M selection. 
the most central $0-1 \%$ events (with $b_{\text {impact }}<1.5 \mathrm{fm}$ ) are considered as a signal, all other events as a background. Purity is defined as a fraction of events, selected by classifier, for which impact parameter is indeed $<1.5 \mathrm{fm}$. The challenge is to increase the purity of $0-1 \%$ selected events. The same set of five descriminating features was used.

Figure 6 (a) shows signal efficiency $v s$ background rejection for a large set of classifiers, probed using TMVA package. It can be seen, that, except for simple cut-based classifiers, all estimators provide similar performance, with slightly better results for neural networks (MLP) and boosted decision trees. Pad (b) of the same figure presents purity in terms of impact parameter in 0-1\% centrality class. The purity level of $\approx 0.53$ (red star on the plot) is obtained for events with conventional V0M selection, while best ML classifiers show values $\approx 0.66$, showing increase in purity by about $13 \%$. This increase is expected since classifiers use information from 5 distinct detecting systems, not just from two VZERO scintillators.

The same classification task was repeated with an extended set of variables (three additional features, mentioned in section 3 , are event-average transverse momentum $\left\langle p_{\mathrm{T}}\right\rangle, \mathrm{K} / \pi$ and $\mathrm{p} / \pi$ ratios). Feature importance histogram is shown in figure 7 , it can be seen that the additional features do not contribute to performance improvement much. Of course, dependence of the results on $\left\langle p_{\mathrm{T}}\right\rangle$ and particle ratios strongly depend the event generator used for the study, so these features may still be interesting if this approach will be applied to real data.

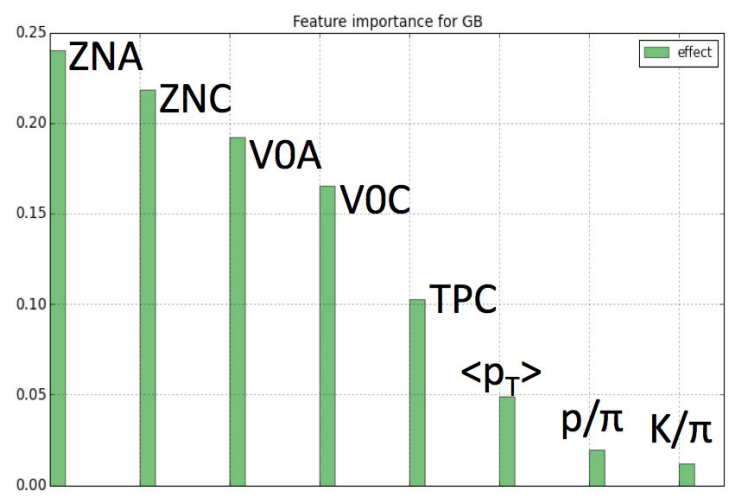

Figure 7. Feature importance for BDTG in classification task for selection of events in $0-1 \%$ centrality class.

\section{$4 \mathrm{ML}$ task for simulated $\mathrm{p}-\mathrm{Pb}$ events}

In proton-nucleus collisions, observed multiplicities and other phenomena are much stronger correlated with $N_{\text {part }}$ than in $\mathrm{Pb}-\mathrm{Pb}$ collisions, while the impact parameter $b$ is only loosely correlated to $N_{\text {part }}$. Therefore, $N_{\text {part }}$ is a more reliable target for machine-learning classification challenge for $\mathrm{p}-\mathrm{Pb}$ collisions than $b$. Let us split truth $N_{\text {part }}$ distribution and the output from some trained ML estimator into 5 classes $0-20,20-40,40-60,60-80,80-100 \%$ (0-20\% - for the most central events). Let us also state ML classification task for $\mathrm{p}-\mathrm{Pb}$ collisions as follows: what is the purity of $0-20 \%$ class selected by ML estimator? In other words, what is the fraction of events, selected by estimator, which indeed belong to the truth $0-20 \%$ class of the highest values of $N_{\text {part }}$ ?

Scikit-learn package [11] was used for this task. Training and testing were done using $5 \mathrm{mln}$ simulated AMPT events of $\mathrm{p}-\mathrm{Pb}$ collisions. Again, charged particles for analysis were taken directly from the generator, without simulation of the detector response. At first, only two features were used in the task, which correspond to "measurements" in Pb-fragmentation direction: multiplicity in VZERO-A scintillator (denoted here as V0A) and number of neutrons-spectators in ZDC-A (denoted as ZNA). Figure 8 (a) is a two-dimensional plot, where scattered points visualize signals in these 
two detectors (a small part of the whole generated event-sample is shown), and decision boundaries, obtained after training of linear discriminant classifier, which drawn by colored areas. Similar plot is in pad (b) for quadratic discriminant classifier. Note that decision boundaries between centrality classes are not drawn by hand, as in ZEM-ZDC histogram from figure 1 (b), but instead are calculated as optimal borders between classes. In the last pad (c) in figure 8, the boundaries are shown for two another features taken for the classification task - multiplicity in VZERO-A and averaged momentum $\left\langle p_{\mathrm{T}}\right\rangle$ of particles in TPC.

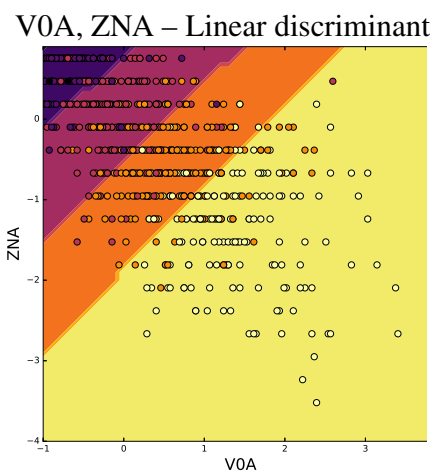

(a)

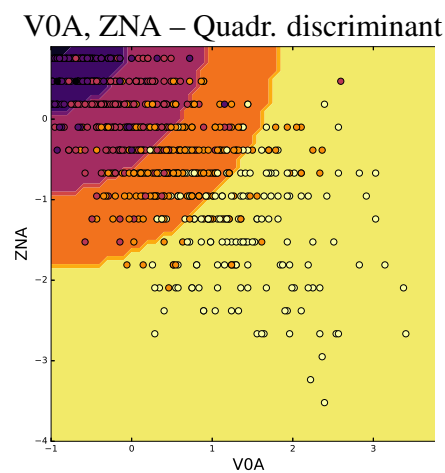

(b)

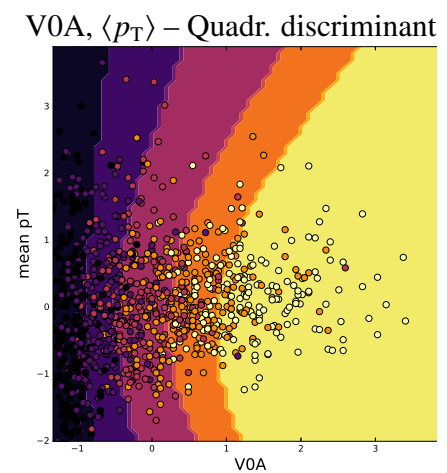

(c)

Figure 8. $2 \mathrm{D}$ plots with the following variables on axes: V0A and ZNA in pads (a, b) and V0A and $\left\langle p_{\mathrm{T}}\right\rangle$ in pad (c). Features are standardized (centered to the mean and scaled to unit variance). Scattered points represent a small fraction of the whole statistics. Decision boundaries are drawn for five centrality classes determined by classifiers: by linear disriminant (a) and by quadratic discriminant (b, c).

What are the purities of classification with different combination of features? For figure 9, the quadratic discriminant was taken as a classifier. Blue circles indicate purity in 0-20\% class of events selected by only one feature: V0A (first point) or ZNA (second point), giving similar values $\approx 0.72$. For each of the next three points, one additional feature was included in ML task, namely, mean momentum $\left\langle p_{\mathrm{T}}\right\rangle$ or multiplicity within TPC. It can be seen, that both these additional features are

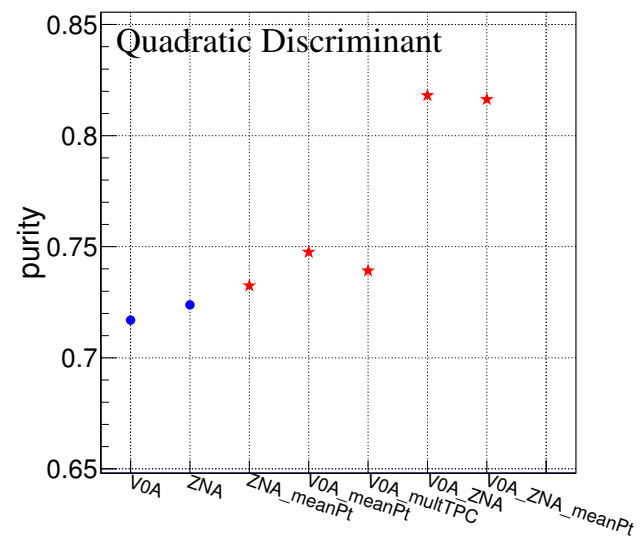

Figure 9. Purity in 0-20\% centrality class with Quadratic discriminant classifier, for several combinations of features. 
weak and provide minimal increase in purity, by $\sim 2-3 \%$. At the same time, when two strong features $\mathrm{V} 0 \mathrm{~A}$ and ZNA are combined, purity rises by $10 \%$ to $\approx 0.82$. A similar behaviour of the purity is obtained for several other classifiers $-k$ nearest neighbours $(k \mathrm{NN})$ with different number of $k$ and for the linear discriminant, see figure 10 (a). Pad (b) of this figure shows purities of 0-20\% class obtained by using single-feature classification (blue circles) in comparison with the best ML result with many features (red star).

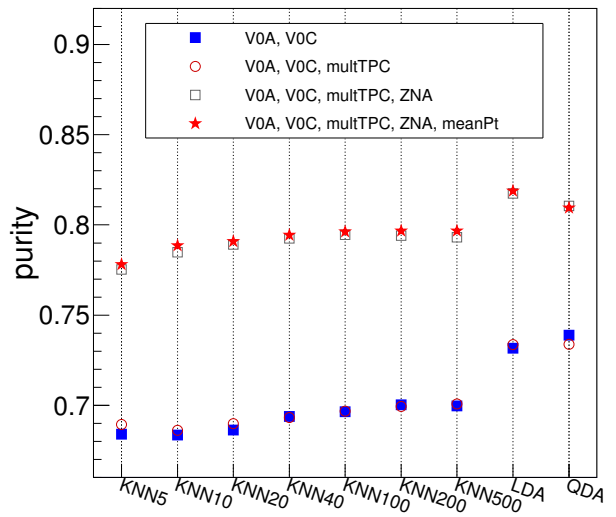

(a)

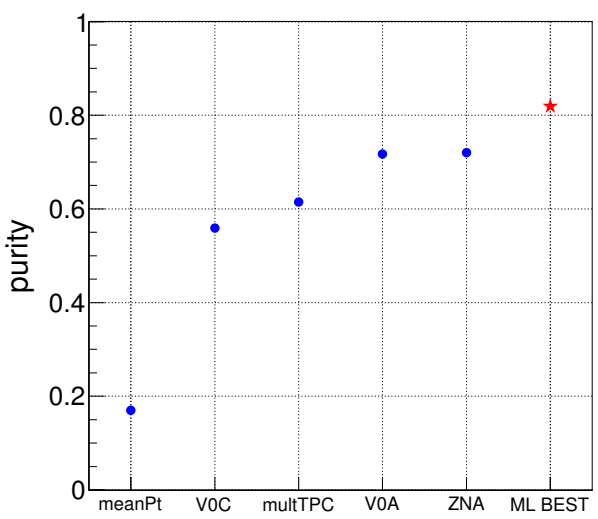

(b)

Figure 10. (a): Purities of $0-20 \%$ centrality class, reached with $k N N$, LDA and QDA classifiers for several feature configurations. (b): Purities obtained with single-feature classification, in comparison with best ML result with many features.

\section{Possible application of ML estimators in NA61/SHINE experiment}

ML-based classifiers can potentially be useful for centrality estimation in fixed target experiments, such as NA61/SHINE experiment at CERN [13]. In that experiment, the Projectile Spectator Detector (PSD) has a modular structure shown in figure 11 and measures the energy of projectile spectators in A-A collisions. Centrality is determined by energy in modules of PSD. Possible improvement can be achieved if the PSD is used in combination with data from several TPC's, and the machine-learning techniques can be applied for that. Moreover, it may be useful to utilize energy deposition in the PSD module-by-module, and try to benefit from all intrinsic correlations between modules. Unfortunately, training of ML estimators requires very robust MC simulations of the PSD. Situation is complicated by the fact that modules in the PSD are fired not only by spectators, but also by particles born in A-A collision. Possibly, methods of unsupervised learning could be adopted for this challenge.

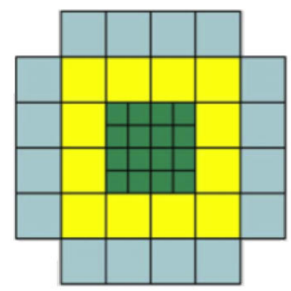

Figure 11. Modular structure of the Projectile Spectator Detector of the NA61/SHINE facility [13] . 


\section{Conclusions}

Accurate centrality determination is a baseline for many physics analyses in heavy-ion collision experiments, for example, for fluctuations and correlations studies of A-A and $\mathrm{p}-\mathrm{A}$ collisions. In the presented exploratory work, we tried to increase resolution of centrality classes in terms of the impact parameter $b$ and the number of nucleons-participants by using machine-learning techniques, which utilizes signals from several detector subsystems simultaneously. Compared with conventional centrality estimators, ML-based estimators allow increasing the resolution and thus the "purity" of centrality classes without losses in statistics (i.e., each centrality class contains the same number of events, but with higher purity). It could be seen that improvement in centrality resolution was achieved especially for the most central events, with increase in purity $\sim 10-13 \%$ compared to conventional centrality selection methods.

If centrality selection is performed using cuts on the signal from a single detecting system, results are obviously worse than if combination of signals from several subdetectors is used. At the same time, simultaneous usage of just two "strong" features may be already enough to significantly increase the purity of centrality class, while additional "weak" features provide very moderate improvement. ML classifiers allow to draw optimized decision boundaries between centrality classes, and obtained boundaries can have non-trivial shapes. It can be especially useful in a multidimensional case when more than two features are used, which would make manual parameterization of decision boundaries very challenging.

Since the presented work is based on simulations, efficiency of the "detectors" is taken to be unity and contamination by secondary particles is zero, which makes some features unrealistically strong. For instance, the "ZDC", used in this study, ideally counts all the neutrons-spectators, which is not the case at all in a real experiment (real ZDC usually has a finite resolution, it loses some neutrons due to combination of spectators into nuclei fragments, and suffers from contamination by non-spectators).

Additionally, there was no attempt made to incorporate intrinsic correlations between selected features, while these correlations are highly dependent on the physics model of the chosen event generator. Also, no attempt was made to train and use ML-based estimators for centrality selection in real data, since it is not easy at all to tune event generator and simulated detector response to match data from real detector. Application of the ML-based centrality estimators to real data needs further investigation.

\section{Acknowledgements}

This work is supported by the Russian Science Foundation, GRANT 16-12-10176.

\section{References}

[1] CMS and LHCb Collaborations, Nature 522, 68-72 (2015)

[2] T. Likhomanenko et al., J. Phys.: Conf. Ser. 664, 082025 (2015)

[3] M. Floris, DataScience@LHC2015 Workshop (report), http://indico.cern.ch/event/395374/ contributions/939892/ (2015)

[4] J. Adam et al. (ALICE Collaboration), Eur. Phys. J. Plus 131168 (2016)

[5] B. Abelev et al. (ALICE Collaboration), Phys. Rev. C 88, 044909 (2013)

[6] M. Miller et al., Ann. Rev. Nucl. Part. Sci. 57, 205 (2007)

[7] K. Aamodt et al. (ALICE Collaboration), Physics Letters B 708, 249-264 (2012)

[8] B. Abelev et al. (ALICE Collaboration), Int. J. Mod. Phys. A 29, 1430044 (2014) 
[9] Zi-Wei Lin et al., Phys.Rev. C 72, 064901 (2005)

[10] A. Hoecker et al., PoS A CAT 040 (2007)

[11] F. Pedregosa et al., JMLR 12, 2825-2830 (2011)

[12] J. Adam et al. (ALICE Collaboration), Phys. Rev. C 91, 064905 (2015)

[13] N.Abgrall et al. (NA61/SHINE Collaboration), JINST 9, P06005 (2014) 